\title{
Social Distance Detection using YOLO
}

\author{
M.Dhivyashree ${ }^{1}$, Abishek Rajendiran ${ }^{2}$, J. Balaji ${ }^{3}$, K.K.Dayanithee Balaji ${ }^{4}$ \\ ${ }^{1}$ Assistant Professor, Department of Computer Science and Engineering, Sri Ramakrishna Engineering College \\ Coimbatore, India \\ ${ }^{2,3,4}$ Student, Department of Computer Science and Engineering, Sri Ramakrishna Engineering College \\ Coimbatore, India
}

\begin{abstract}
The COVID-19 (Corona virus) has sparked global panic after spreading to over 180 countries and causing $3,519,901$ confirmed cases compared to 247,630 passing globally as of May 2020.The lack of any complex helpful experts, and therefore the need for resistance to COVID19, increases the population's defenselessness. Since there are no medicine available to cure, social distancing is the only viable option for combating this infection.Pre-trained deep neural network models, such as YOLOv3, is being used in this research to detect people with the help of bounding boxes that identify the human beings. The system also analyses the distances amongst people in the society using the Euclidean distance metric approach to estimate the proportion of people who infringe the social distancing within the camera footage.
\end{abstract}

Keywords: COVID-19, YOLO Algorithm, Video surveillance, Social distancing, Pedestrian detection, Pedestrian tracking.

\section{INTRODUCTION}

Social distancing is a lucid infection prevention and control intervention implemented to avoid contact between those that are infected with a disease causing pathogen and people who aren't, so on stop the speed and extent of disease transmission among the group. This results in decrease in spread, morbidity and mortality due to the disease. As of now many healthcare organizations has put forth their contribution in the development of vaccine and successfully they created the vaccine against this deadly virus, but still people has to make sure that they are following the advisable social distance of 2 metre as the fatality rate is increasing day-by-day. This circumstance enforces the global community to appear for alternate ways to prevent the spread of this infectious virus. Social distancing is claimed as the best spread stopper within this deadly situation, and every affected countries are locked-down to implement social distancing. This research is aimed to diminish and allay spread of virus among the people. The world has not yet fully endure this pandemic and still the virus is mutating into deadlier than before. However, to scale back the impact of the pandemic on the country's economy, several governments have allowed a limited number of economic activities to be resumed once the quantity of recent cases of Covid-19 has dropped below a threshold level. As these countries cautiously restarting their economic activities, concerns have emerged regarding workplace safety within the environment. To cut back the chance of infection, it is highly advised that people should avoid any person-to-person contact like shaking hands and that they should maintain a distance of a minimum of 1 meter from one another.

\section{RELATED WORKS}

As per various health standards [3] it is highly recommended that people should maintain a minimum of 2 meters of distance among each other so as to follow social distancing in public places. A recent study stipulates that social distancing is a sensible containment measure and essential to pre-empt SARSCoV-2 among the populace, because people with mild or no symptoms may coincidentally carry infection and may infect other people [4]. The other study accumulated on detecting the mode of its transmission in order to lower the spread among the people worldwide. The study, with the assistance of ferrets, showed that SARS-CoV-2 can be transmitted easily via any kinds of direct contact and through the droplets of sneeze by the person as well. The ferrets may be seen getting infected within a week after being exposed to the virus. The study resulted experimental proof of the virus transmission to be airborne, hence, supporting the implementation of social distancing among the crowd that is being currently practiced in various countries all over the world. Respiratory diseases are infectious and highly contagious where the speed and mode of transmission of the causing virus are the essential factors to be considered for the treatment or ways to put off the spread of the virus within the community and people should not jostle in any public events in the society. Several medicinal organizations and researchers had worked and developed the vaccine for COVID-19, but still, there's no well-known medicine available for treatment. Therefore, the preventative measures and precautionary concerns are taken by the nations across the world 
DOI: $10.17148 /$ IARJSET.2021.8587

to cut off the rate of infection. Within a month, the upsurge in China gained an epitome within the first week of February with around 4,000 new confirmed cases each and every day. For the first time after this pandemic, there are a testament of ease with no new confirmed cases for five succeeding days till 23 March 2020 [14].This is apparent that social distancing measures followed in China initially and adopted worldwide later to control COVID-19 virus [18].In Consideration of novel coronavirus outbreak began, many nations are taking the succour of technology based solutions in different capacities to contain the pervasive corona virus [19], [20]. Various developed and developing nations, including India and Asian nation, for case, utilizing GPS to trace the movements of the suspected or infected persons to observe any chance of their revelation among healthy people. In India, the government is using the Arogya Setu App, which worked with the aid of GPS and Bluetooth to point down the existence of COVID-19 patients within the locality that differs from place-to-place. It also suggests everyone to stay an advisable distance from the infected person [21].

\section{METHODOLOGY}

This technique for detecting social distancing was created to detect the safety distance between people in shared spaces. During this research, the deep CNN approach and computer vision techniques are being used. Originally, the pedestrian in the video frame was spotted using an open-source object detection approach that relies on the YOLOv3 algorithm. Only the pedestrian class was used in the detection analysis, and other object classes were neglected throughout this application. As a result, within the image frame, the bounding box that most perfectly aligns each detected pedestrian is depicted, and these results of observed commuters will be used for inter-distance calibration. In main camera, the camera is captured at a specific potential as a video frame, and the video frame is then processed into a two-dimensional top-down view for far more reliable data acquisition. The pedestrians in the video frame are presumed to be walking on about the same plane throughout this methodology. The top-down view is provided by extracting four featured plane points from the image. The top-down view can then be used to compute the position about each pedestrian. Pedestrian distances are too often monitored and configured. Any distance lower than the permissible distance between any two individuals is illustrated with red lines that serve as preventative caution, based on the pre-specified minimum distance. The entire app was then fed into the tkinter GUI. The experiment has been carried out using the Python programming language. Figure 1 depicts the flowchart of the mechanism for the social distancing detection tool.

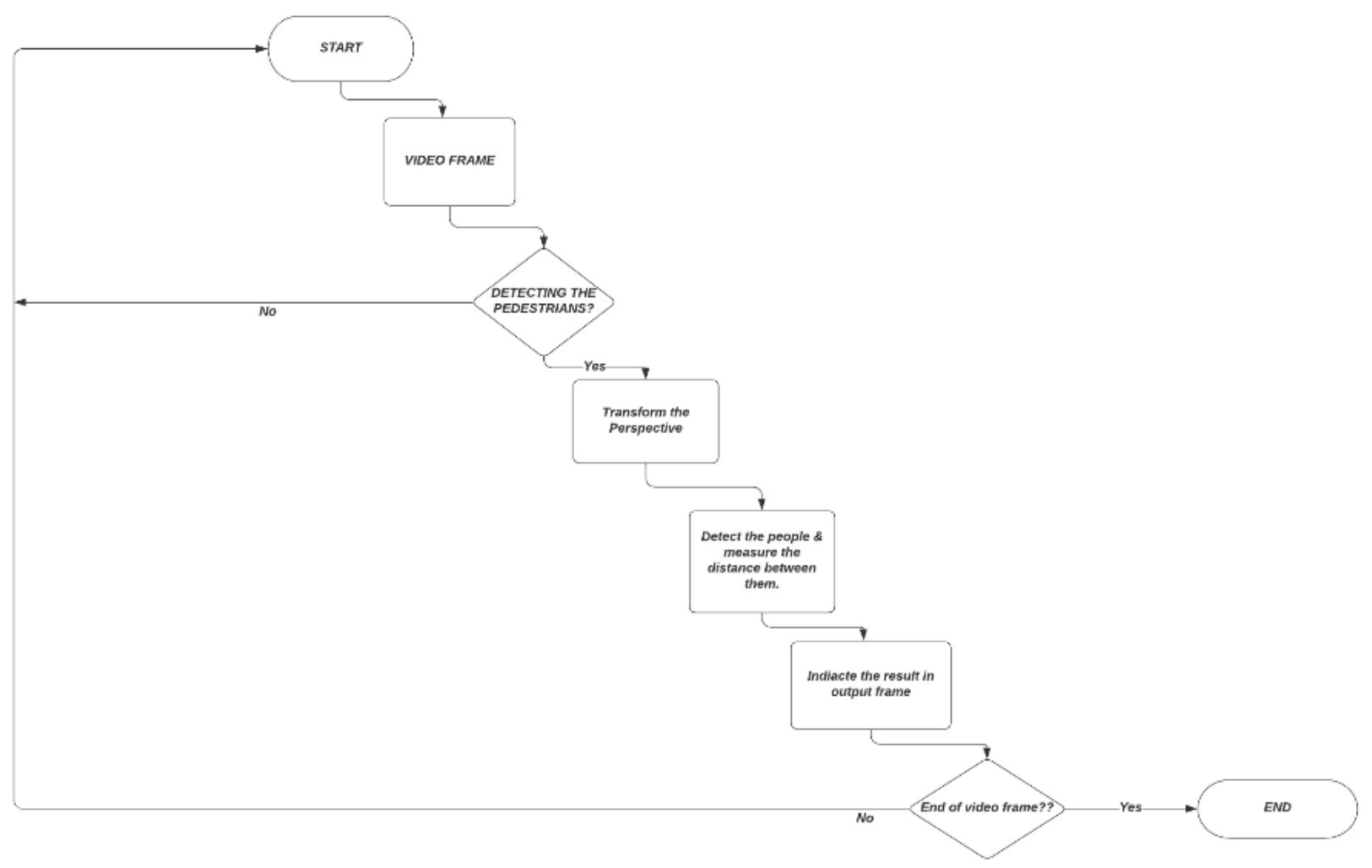

Figure 1 Proposed block diagram 


\section{International Advanced Research Journal in Science, Engineering and Technology}

Vol. 8, Issue 5, May 2021

DOI: $10.17148 /$ IARJSET.2021.8587

\section{A. Pedestrian detection using YOLO}

Deep CNN framework is proposed as an object detection technique that exacerbated dimensionality apprehensions by structuring detection as a single regression problem [11]. When it relates to deep learning-based object detection, the YOLO model can be found one of the state-of-the-art object detectors which is shown to have spectacular performance perks and is suitable for real-time use. Figure 2 illustrates the YOLO model for pedestrian detection that was actively supported throughout this research. The YOLO algorithm was conceived as an object detection algorithm that acquired bounding box coordinates $(\mathrm{x}, \mathrm{y}, \mathrm{w}, \mathrm{h})$, object confidence, and corresponding class labeling probabilities $\left(\sum_{1}^{\mathrm{n}} \mathrm{P}\right)$ while learning bounding box coordinates $(\mathrm{x}, \mathrm{y}, \mathrm{w}, \mathrm{h})$. The YOLO has been trained on the COCO dataset, which seems to have 80 labels in whole, including human and pedestrian gatherings. For pedestrian detection in this experiment, the YOLO model's main box coordinates, object confidence, and pedestrian object classification from the detection point are included.

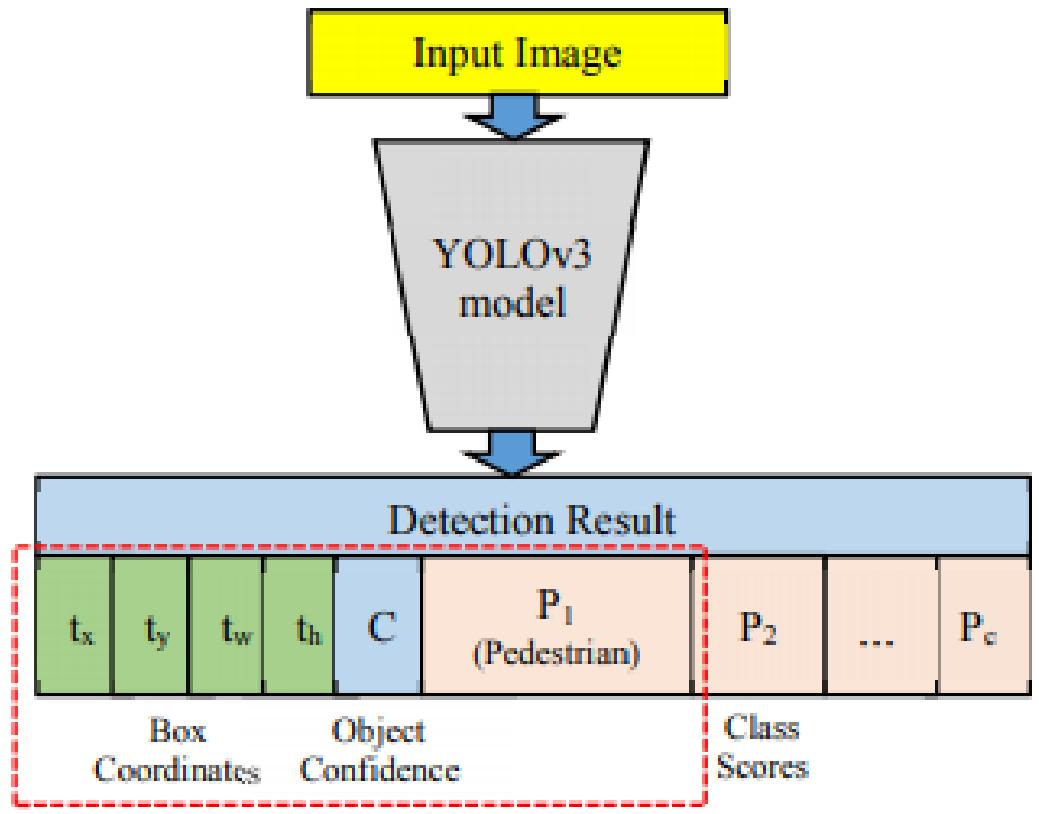

The parameters used for pedestrian detection

Figure 2 YOLO model for people detection

\section{B. Distance measurement using Euclidean method}

The current position of the bounding box for each individual ( $\mathrm{x}, \mathrm{y}, \mathrm{w}, \mathrm{h}$ ) inside the perspective view is detected and converted into a top-down view in this step of this process. The location of every pedestrian in the top-down view is assessed by holding up the bottom-center point of the bounding box. The distance between each pedestrian pairs has often been assessed from a top-down perspective, and indeed the distances are scaled utilizing scaling factor estimated from camera view measurements. Given the positions of two pedestrians in an image as (x1, y1) and (x2, y2), the variance between them, $\mathrm{d}$, will be determined as:

$$
\text { Distance }=\sqrt{\left(\mathrm{x}_{2}-\mathrm{x}_{1}\right)^{2}+\left(\mathrm{y}_{2}-\mathrm{y}_{1}\right)^{2}}
$$

The pedestrians whose distance is still less than the minimum recommended distance, min, are highlighted in red, while the remaining portion are highlighted in green. The coloring saturation operation of every bounding box, c, can be represented as:

$$
\mathrm{c}=\left\{\begin{array}{l}
\text { red }, \text { distance }<\min \\
\text { green }, \text { distance } \geq \min
\end{array}\right.
$$

\section{GUI Creation using Tkinter}

Tkinter is the pre - compiled GUI archive, which can be seen in Figure 3. Python and Tkinter provide such a fast and efficient way to design graphical user interfaces. Tkinter offers the Tk GUI toolbox a robust object-oriented interaction. 
It's quick to manage a GUI configuration with Tkinter. Belatedly our whole features and functions are injected into tkinter GUI for the user-friendliness usage.

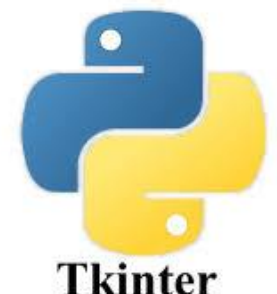

Figure 3 tkinter Library

\section{RESULT AND DISCUSSION}

The pedestrian is seen walking down a public street in the video, which is associated to the application in Figure 5. The video frame in this process is fixed to the sidewalk at some angle. For a more reliable distance calculation, the video frame's points of view is modified into a top-down view. The homepage of the application is displayed in Figure 4.The observation of social distancing in a video frame and the implications of the top-down view could be seen in Fig 6 . From top to bottom, the sequences are shown. For the purpose of checking social distancing, the points reflect almost every pedestrian. The pedestrians whose distance from some other pedestrian is less than the appropriate threshold are indicated by red markers, while pedestrians who maintain a considerable distance from those other pedestrians are indicated by green points. However, as seen from the images below, there are a number of detection errors. The pedestrians walking too near as possible to another pedestrian before they are overlaid on the camera view is perhaps the factor of these errors. The YOLO algorithm could therefore detect the pedestrian's body as an entity by expressing the bounding box; the pedestrian's orientation corresponds to the middle-point of the bounding boxes, which is more credible. The processed result, that's a number of violations, is compared in Fig 7 over the video frame, it will later be kept into our system, where we'll be able to utilize it several times as we need.

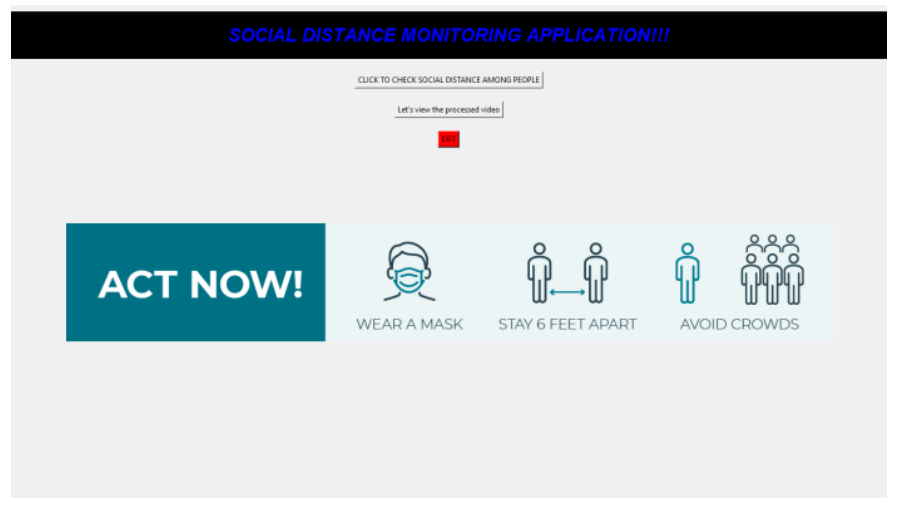

Figure 4 Homepage of the application

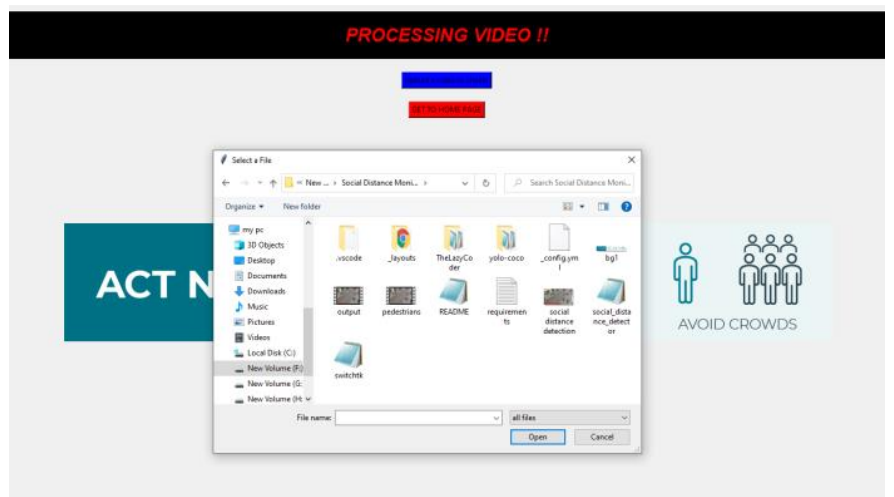

Figure 5 Browsing a video for monitoring 


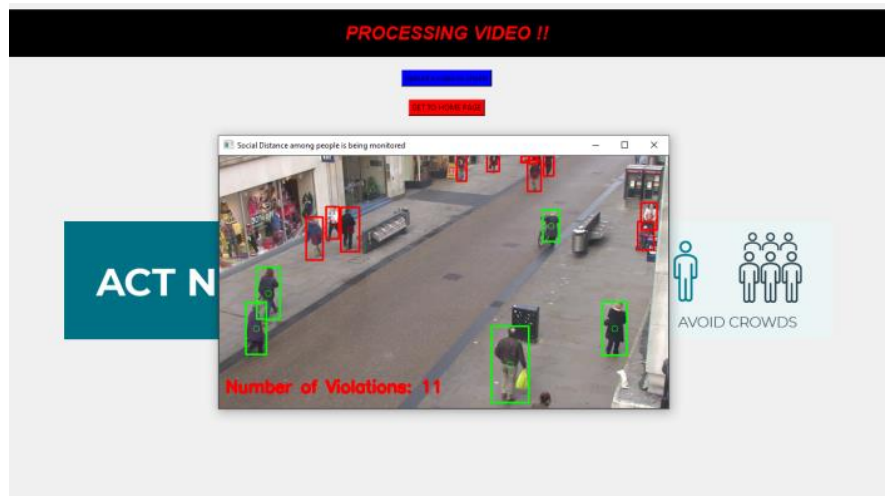

Figure 6 Input video is being monitored

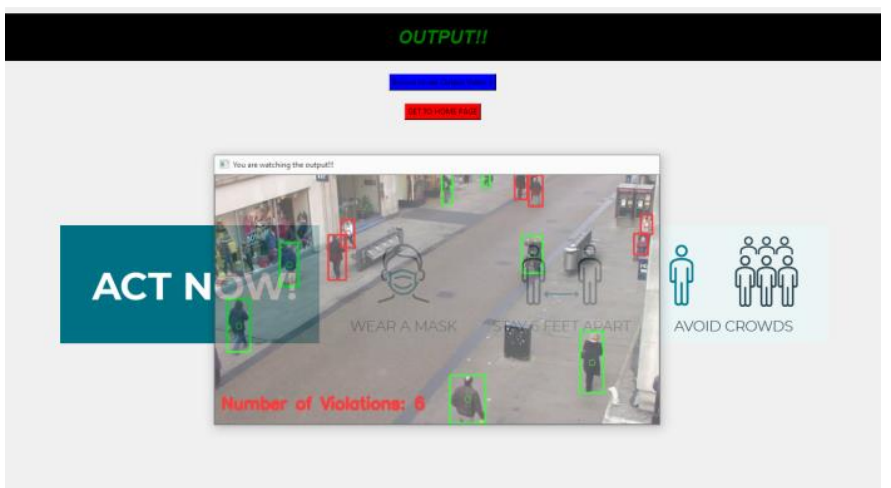

Figure 7 Viewing the processed video stored in the system

\section{CONCLUSION AND FUTURE WORK}

A deep learning model is now used to develop a means of detecting social distancing. The distance between people will be inferred using computer vision, and any uncommon pair of individuals will be indicated with a red frame and a line. A footage of pedestrians crossing the street is being used to test the proposed process. The visual results confirmed that the proposed model is effective in monitoring social distancing measures between groups of people, and this could be further incorporated for use in other settings such as hospitals, campuses, industries, and establishments. Plenty more algorithms with higher accuracy and robustness will be conducted in the future, allowing the system to gradually improve. This application can also be integrated in any security camera to monitor people in real life time.

\section{REFERENCES}

[1]. M. Andriluka, S. Roth, and B. Schiele, "People-tracking-by-detection and people-detection-by-tracking," in 2008 IEEE Conference on computer vision and pattern recognition. IEEE, 2008, pp. 1-8.

[2]. N. S. Punn and S. Agarwal, "Crowd analysis for congestion control early warning system on foot over bridge," in 2019 Twelfth International Conference on Contemporary Computing (IC3). IEEE, 2019, pp. 1-6

[3]. L. Hensley, "Social distancing is out, physical distancing is in - here's how to do it," Global News-Canada (27 March 2020), 2020.

[4]. ECDPC, "Considerations relating to social distancing measures in response to COVID-19 secondupdate,"https://www.ecdc.europa.eu/en/publications-data/considerations, 2020, [Online; accessed March 23, 2020].

[5]. D.-Y. Chen, C.-W. Su, Y.-C. Zeng, S.-W. Sun, W.-R. Lai, and H.- Y. M. Liao, "An online people counting system for electronic advertising machines," in 2009 IEEE International Conference on Multimedia and Expo. IEEE, 2009, pp. 1262-1265

[6]. C.-W. Su, H.-Y. M. Liao, and H.-R. Tyan, "A vision-based people counting approach based on the symmetry measure," in 2009 IEEE International Symposium on Circuits and Systems. IEEE, 2009, pp. 2617-2620

[7]. B. Leibe, E. Seemann, and B. Schiele, "Pedestrian detection in crowded scenes," in 2005 IEEE Computer Society Conference on Computer Vision and Pattern Recognition (CVPR'05), vol. 1. IEEE, 2005, pp. 878-885.

[8]. Z.-Q. Zhao, P. Zheng, S.-t. Xu, and X. Wu, "Object detection with deep learning: A review," IEEE transactions on neural networks and learning systems, vol. 30, no. 11, pp. 3212-3232, 2019.

[9]. M. Putra, Z. Yussof, K. Lim, and S. Salim, "Convolutional neural network for person and car detection using yolo framework," Journal of Telecommunication, Electronic and Computer Engineering (JEC), vol. 10, no. 1-7, pp. 67-71, 2018

[10]. N. Wojke, A.Bewley, and D.Paulus,"Simple online and realtime tracking with a deep association metric," in 2017 IEEE international conference on image processing (ICIP). IEEE,2017,pp. 3645-3649.

[11]. Wu, J., Tang, B., Bragazzi, N., Nah, K. and McCarthy, Z., 2020. Quantifying the role of social distancing, personal protection and case detection in mitigating COVID-19 outbreak in Ontario, Canada. Journal of Mathematics in Industry. 


\title{
International Advanced Research Journal in Science, Engineering and Technology
}

\author{
Vol. 8, Issue 5, May 2021
}

\section{DOI: $10.17148 /$ IARJSET.2021.8587}

[12]. A. Brunetti, D. Buongiorno, G. F. Trotta, and V. Bevilacqua, "Computer vision and deep learning techniques for pedestrian detection and tracking: A survey," Neurocomputing, vol. 300, pp. 17-33, 2018

[13]. R. Girshick, J. Donahue, T. Darrell, and J. Malik, "Rich feature hierarchies for accurate object detection and semantic segmentation," in Proceedings of the IEEE conference on computer vision and pattern recognition, 2014, pp. 580-587

[14]. N. H. C. of the People's Republic of China, "Daily briefing on novelcoronavirus cases in China,"http://en.nhc.gov.cn/202003/20/c78006.htm, 2020, [Online; accessed March 20, 2020].

[15]. J. Redmon, S. Divvala, R. Girshick, and A. Farhadi, "You only look once: Unified, real-time object detection," in Proceedings of the IEEE conference on computer vision and pattern recognition, 2016, pp. 779-788

[16]. S. Ren, K. He, R. Girshick, and J. Sun, "Faster r-cnn: Towards real-time object detection with region proposal networks," in Advances in neural information processing systems, 2015, pp. 91-99.

[17]. Merkulova, I., Shavetov, S., Borisov, O. and Gromov, V., 2019. Object detection and tracking basics: Student education. IFAC-PapersOnLine, 52(9), pp.79-84

[18]. S. K. Sonbhadra, S. Agarwal, and P. Nagabhushan, "Target specific mining of covid-19 scholarly articles using one-class approach," 2020

[19]. N. S. Punn and S. Agarwal, "Automated diagnosis of covid-19 with limited posteroanterior chest X-ray images using fine-tuned deep neural networks," 2020.

[20]. N. S. Punn, S. K. Sonbhadra, and S. Agarwal, "Covid-19 epidemic analysis using machine learning and deep learning algorithms," medRxiv, 2020.[Online].Available:https://www.medrxiv.org/content/ early/2020/04/11/2020.04.08.20057679

[21]. O. website of Indian Government, "Distribution of the novel coronavirus-infected pneumoni Aarogya SetuMobileApp,"https:// www.mygov.in/aarogya-setu-app/, 2020.

[22]. Varshaa a; Vinitha V; Usha Nandhini D; Yogeshwaran R; Soundharya B M. "Artificial intelligence and its applications- A Review". International Research Journal on Advanced Science Hub, 1, 2, 2020, 1-4. doi: 10.47392/irjash.2019.11

[23]. Kousalya M.; Lakshi E.; Mukesh Kanna R.K.; Pravin G.; Prabhu T.. "Automatic Restaurant Food Ordering Menu Card". International Research Journal on Advanced Science Hub, 3, Special Issue ICARD-2021 3S, 2021, 7-12. doi: 10.47392/irjash.2021.052

[24]. Jayavarthini S; Savitha V; Mathanraj K; Nandha kumar P; Sangeetha K. "On road assistant finder". International Research Journal on Advanced Science Hub, 3, Special Issue ICARD-2021 3S, 2021, 18-22. doi: 10.47392/irjash.2021.054

[25]. Pranesh S; Jenita J R; Nisha D; Prabakar D. "Sentimental Data Analysis-To Predict the User Emotions". International Research Journal on Advanced Science Hub, 3, Special Issue ICARD-2021 3S, 2021, 26-29. doi: 10.47392/irjash.2021.056 